Miami Nature Biotechnology Short Reports

TheScientificWorld (2001) 1(S3), 86SR

ISSN 1532-2246; DOI 10.1100/TSW.2001.115

\title{
LIPOTOXIC NEPHROPATHY IS MORE SEVERE IN MALE THAN FEMALE RATS N WITH NON-INSULIN DIABETES MELLITUS (NIDDM)
}

\author{
Jesus H. Dominguez*, Wei Xu, John W. Hawes , and Richard G. Peterson \\ Department of Medicine, Biochemistry, and Anatomy: IUMC and VAMC, Indianapolis, IN, \\ 46202, USA \\ * jhdoming@iupui.edu
}

INTRODUCTION. Dyslipidemia is a prominent risk factor for nephropathy in NIDDM; as indicated by longitudinal studies $(1,2)$. This association is not surprising, as the most severe examples of hypercholesterolemia are either familial, or caused by NIDDM (3). Moreover, dyslipidemia in NIDDM is also coupled with markedly increased levels of oxidized LDL (oxLDL) (4), and heightened oxidative stress is recognized to complicate NIDDM (5). However, specific renal toxicity of lipid oxidant (lipotoxicity) is not proven in NIDDM, perhaps because such proof is difficult to obtain in human investigations. We tested the hypothesis that lipotoxicity enhances the level of glomerular oxidized LDL receptor 1 (OLR1) in obese males rats with NIDDM, augmenting oxidant stress and resulting fibrosis.

METHODS. First generation $\left(\mathrm{F}_{1}\right)$ hybrid rats derived from the Zucker fatty diabetic strain (ZDF) and the spontaneous hypertensive heart failure rat (SHHF/Gmi-fa) were studied for 41 weeks on two diets. Three groups of rats, 10 obese males (OM5008), 13 obese females (OF5008), and 10 lean males (LM5008), were fed diet \# 5008 (27 \% protein, $17 \%$ animal fat, $56 \%$ carbohydrate, and 11.1 uMoles/gm lipid hydroperoxide). Two other groups, 3 OM12470 and 14 OF12470, were aging controls, and ate diet \#12470 (10 \% protein, $48 \%$ animal fat, 42 \% carbohydrate, and 4.5 uMoles/gm lipid hydroperoxide).

RESULTS. OM5008 rats had an atherogenic plasma lipid profile, with markedly elevated plasma cholesterol and hydroperoxides. Their glomeruli expressed OLR1 (antibody from Dr. M. Nagase) and accumulated 4-OH-nonenal (4HNE); the highly cytotoxic lipid peroxide endproduct with mitochondria localization. OM5008 also had the highest levels of creatinine, renal Transforming Growth Factor $\beta$-1 (TGF $\beta$-1) and glomerulosclerosis. Remarkably, hypertension and hyperglycemia were not directly linked to renal injury.

\begin{tabular}{|c|c|c|c|c|c|c|}
\hline GROUP & $\begin{array}{l}\text { Cholesteml } \\
\text { (mH) }\end{array}$ & $\begin{array}{l}\text { TBARS } \\
\text { (UW) }\end{array}$ & $\begin{array}{l}\text { Glomerular } \\
\text { OLFil }{ }^{*} \text { ) }\end{array}$ & $\begin{array}{l}\text { Fenal 4HWE } \\
{[* \text { ] }}\end{array}$ & $\begin{array}{l}\text { Plasma } \\
\text { Creatinine (uH) }\end{array}$ & $\begin{array}{l}\text { Fienal TGFBl } \\
\text { Peje prot }\end{array}$ \\
\hline OM50108 (i) & $18.8 \pm 2.9$ & $22 \pm 4$ & $50 \pm 5$ & $2.43 \pm 0.31$ & $100 \pm 16$ & $715 \pm 65$ \\
\hline OF5008 & $7.7 \pm 1.2$ & $12 \pm 1$ & $10 \pm 3$ & $0.58 \pm 0.15$ & $46 \pm 1$ & $290 \pm 72$ \\
\hline L MS008 & $2.2 \pm 0.1$ & $8 \pm 1$ & $6 \pm 2$ & $0.75 \pm 0.06$ & $42 \pm 1$ & $55 \pm 13$ \\
\hline $0 \mathrm{MI} 2470$ & $9.9 \pm 1.4$ & $13 \pm 1$ & $7 \pm 3$ & $1.31 \pm 0.12$ & $50 \pm 3$ & $370 \pm 46$ \\
\hline $0 F 12470$ & $25 \pm 0.2$ & $10 \pm 1$ & $7 \pm 2$ & $0.51 \pm 0.04$ & $41 \pm 1$ & $272 \pm 53$ \\
\hline
\end{tabular}


CONCLUSIONS. Glomerular expression of OLR1 is linked to deposits of 4HNE and glomerulosclerosis. We presume that in OM5008, OLR1 enhances glomerular uptake of oxidized LDL, causing greater oxidant stress, TGF $\beta 1$ production, and glomerulosclerosis. In OF5008, renal protection from oxidants is conferred by blunted glomerular OLR1 expression and an effective renal antioxidant system.

\section{REFERENCES.}

1. $\quad$ 1. $\quad$ Ravid, M., Brosh, D., Ravid-Safran, D., Levy, Z., and Rachmani, R. (1998) Arch. Intern. Med. 11, 998-1004

2. $\quad 2 . \quad$ Yokoyama, H., Okudaira, M., Otani, T., Watanabe, C., Takaike, H., Miuira, J., Yamada, H., Mutou, K., Satou, A., Uchigata, Y., and Iwamoto, Y. (1998) Diabetes Care 21, 1080-1085

3. $\quad 3 . \quad$ Knopp, R.H. (1999) N. Engl. J. Med. 341, 498-511

4. 4. Yoshida, H., Ishikawa, T., and Nakamura, H. (1997) Arterioscler. Thromb. Vasc. Biol. 17, 1438-1446

5. $\quad 5 . \quad$ Delmas-Beauvieux, M.C., Peuchant, E., Thomas, M.J., Dubourg, L., Pinto, A.P., Clerc, M., and Gin, H. (1998) Clin. Biochem. 31, 221-228 


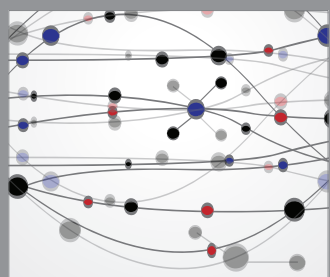

The Scientific World Journal
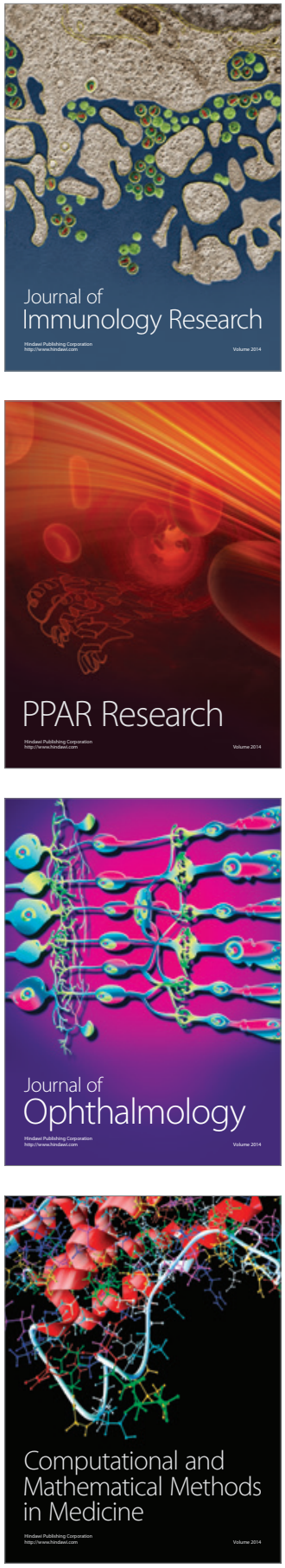

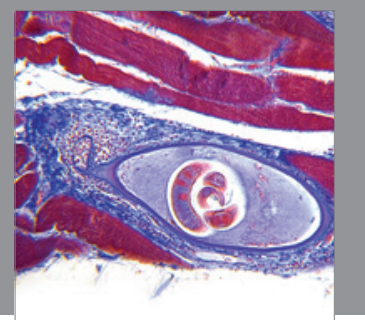

Gastroenterology

Research and Practice
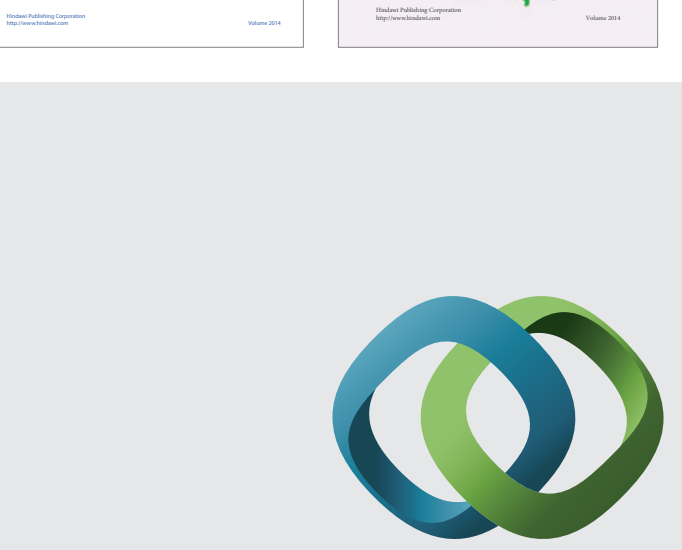

\section{Hindawi}

Submit your manuscripts at

http://www.hindawi.com
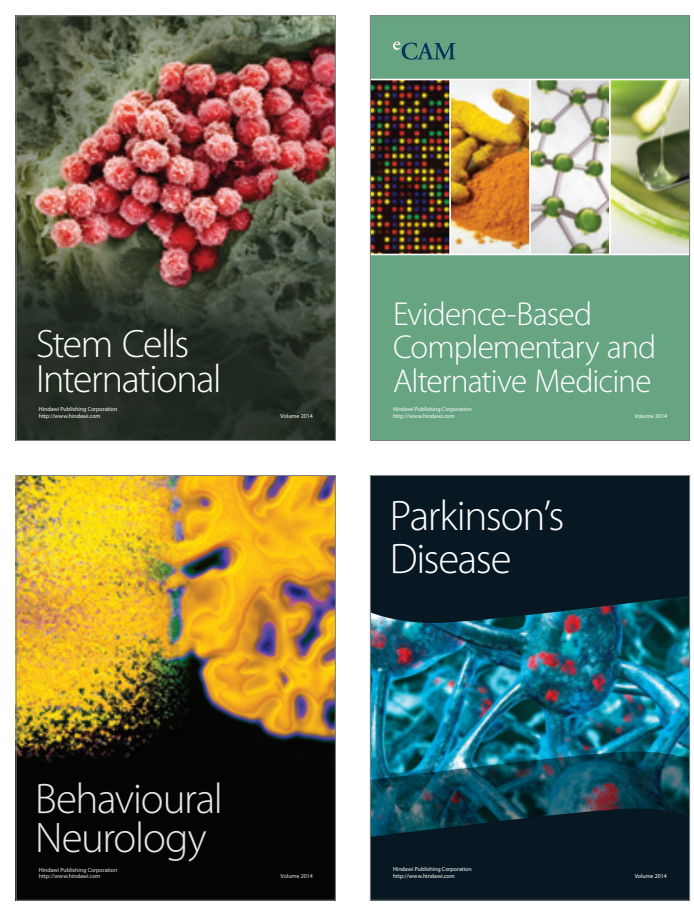

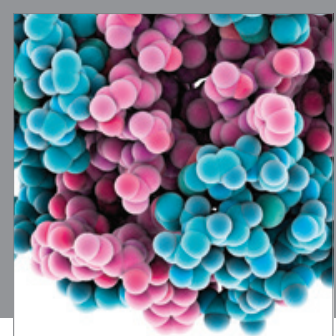

Journal of
Diabetes Research

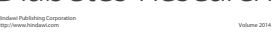

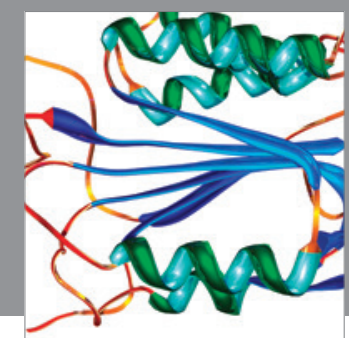

Disease Markers
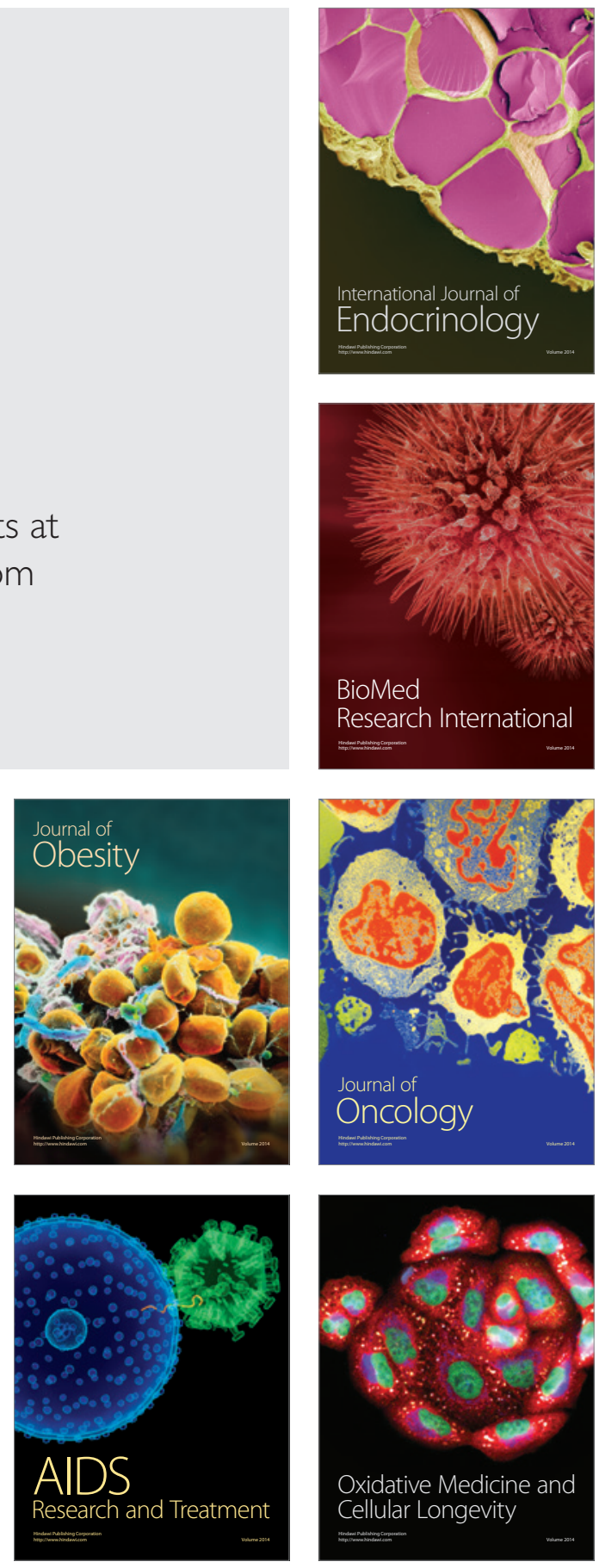